Research Article

\section{The validity of progesterone level on hCG injection day in the prediction of IVF/ICSI cycles' outcome}

\author{
Mostafa A Abolfotouh ${ }^{1,2 *}$, Hallah Al-Anazi ${ }^{3}$ and Samar Hassan ${ }^{1-3}$ \\ ${ }^{1}$ King Abdullah International Medical Research Center, Ministry of National Guard Health Affairs, \\ Riyadh, Saudi Arabia \\ ${ }^{2}$ King Saud bin-Abdulaziz University for Health Sciences, Ministry of National Guard Health Affairs, \\ Riyadh, Saudi Arabia \\ ${ }^{3}$ Reproductive Endocrinology \& Infertility Unit, Obstetrics \& Gynecology Department, Ministry of \\ National Guard Health Affairs, Riyadh, Saudi Arabia
}

\section{Abstract}

Background: Previous studies highlighted the negative effect of premature progesterone elevation (PE) during IVF cycles on the cycle outcomes. The aim of this study was to assess the validity of progesterone level on hCG day $\left(P_{4}\right)$ in the prediction of IVF/ICSI cycles' outcome.

Methods: In a retrospective cohort study, all fresh cycles of 256 patients who underwent IVF or ICSI cycles in 2017 at reproductive endocrinology \& infertility unit/ Obg/Gyn department at King Abdulaziz Medical city, Riyadh, Saudi Arabia, were followed up. They were started on gonadotropin medications for ovarian hyperstimulation, followed by serial transvaginal U/S and serum estrogen levels each visit. Patients having 2 or more $18 \mathrm{~mm}$ follicles were triggered by hCG 10,000 IU and ovum pickup was done 34-36 hrs after. Data were collected on patients' characteristics [age, BMI infertility type], cycles' characteristics [number of follicles and endometrium thickness on hCG day, $P_{4}$ and estrogen levels], rates of pregnancy and pregnancy outcomes. Receiver operating characteristic curve was applied to determine the cut-off of $P_{4}$ that corresponds with a negative pregnancy test. Logistic regression analysis was used and significance was considered at $p$ - value of $\leq 0.05$.

Results: Pregnancy rate in the study sample was $36.7 \%$. The mean $P_{4}$ level in cycles with negative pregnancy tests was significantly higher than the mean in cycles with positive tests $(p=0.018)$. After adjusting for confounders, significant negative association between $P_{4}$ and pregnancy rate was evident $(p<0.03)$. The optimum trade-off of $P_{4}$ for prediction of a negative pregnancy test was $1.5 \mathrm{nmol} / \mathrm{L}$. This cut-off level had a $59 \%$ sensitivity, $51 \%$ specificity and $68 \%$ positive predictive value and $10 \% \& 15 \%$ absolute and relative risk reductions respectively. Cycles with mean $P_{4}$ of $\geq 1.5 \mathrm{nmol} / \mathrm{L}$ were significantly associated with primary infertility $(p=0.011)$, lower mean BMI $(p=0.009)$ higher mean estrogen level $(p<0.001)$, lower live birth rate $(p=0.048)$, higher abortion rate $(p=0.039)$, and higher ovarian hyperstimulation rate $(p=0.027)$.

Conclusion: Premature elevation of progesterone level on the hCG day in IVF/ICSI cycles may have adversely impacted the pregnancy rate and pregnancy outcome. The cutoff point of $1.5 \mathrm{nmol} / \mathrm{L}$ for this $P_{4}$ was not valid in predicting pregnancy outcomes.

\section{More Information}

*Address for Correspondence: Mostafa A Abolfotouh, Professor, Research Training \& Development Section, King Abdullah International Medical Research Center (KAIMRC), King Saud bin-Abdulaziz University for Health Sciences (KSAU-HS), Ministry of National Guard- Health Affairs, Riyadh 11426, Saudi Arabia, P.O. Box 3660, Riyadh 11481, Mail Code 1515 (KAIMRC), Tel: +966 (11) 429-4460; FAX: +966 (11) 4294440; \# 00966503659204 ;

Email: mabolfotouh@gmail.com

Submitted: 04 September 2019 Approved: 10 September 2019 Published: 11 September 2019

How to cite this article: Abolfotouh MA, Al-Anazi $\mathrm{H}, \mathrm{Hassan} \mathrm{S}$. The validity of progesterone level on hCG injection day in the prediction of IVF/ICSI cycles' outcome. Clin J Obstet Gynaecol. 2019; 2: $095-100$

DOI: dx.doi.org/10.29328/journal.cjog.1001028

Copyright: @ 2019 Abolfotouh MA, et al. This is an open access article distributed under the Creative Commons Attribution License, which permits unrestricted use, distribution, and reproduction in any medium, provided the original work is properly cited

Keywords: Pregnancy rate; Premature progesterone rise; Prediction: Validity; Ovarian hyper stimulation; Pregnancy outcome

Abbreviations: hCG: human Chorionic Gonadotropin; PE: Premature Progesterone Elevation; $P_{4}$ : Progesterone level on hCG day; E2: Estrogen level, PMS: Percentage Mean Score; $\mathrm{COH}$ : Hyperstimulation; ROC: Receiver Operating Characteristic Curve; AUC: Area Under the Curve; ARR: Absolute Risk Reduction, RRR: Relative Risk Reduction; HER: Electronic Health Records; KAIMRC: King Abdullah International Medical Research Center; IRB: Institutional Review Board

Check for updates

\section{Background}

In the normal menstrual cycle, progesterone level is rising after luteinizing hormone ( $\mathrm{LH}$ ) surge and ovulation in the luteal phase. In in vitro fertilization (IVF) cycles, progesterone level sometimes rise in the follicular phase on the day of human chorionic gonadotropin (hCG) administration. This premature progesterone rise (PE) was called premature luteinization referred to premature LH surge. With the introduction of GnRH analogues in IVF cycles, the progesterone level $\left(P_{4}\right)$ was decreased and premature LH surge was inhibited. This is because suppressing of granulosa cell steroidogenic activity. 
Despite the wide use of GnRH analogous in ART nowadays, the rise of progesterone level is still observed in some cycles. The effect of that on the endometrium and cycle outcome is still controversial and area for discussion $[1,2]$.

Although the frequency of PE varies, incidences as high as $35 \%$ of stimulated cycles in women treated with $\mathrm{GnRH}$ agonists $[3,4]$ and $38 \%$ of cycles in women treated with $\mathrm{GnRH}$ antagonists [5,6], have been reported. However, in a large retrospective analysis of over 4000 cycles, the incidence of $\mathrm{PE}$ above $1.5 \mathrm{ng} / \mathrm{ml}$ was estimated to be $8.4 \%$ in agonist and antagonist cycles $[7,8]$.

The relationship between PE and pregnancy rate has been analyzed by using different thresholds of serum progesterone on the day of hCG. The thresholds were varied and found to be between $0.4 \mathrm{ng} / \mathrm{ml}$ and $3 \mathrm{ng} / \mathrm{ml}$ [9]. For example, in analysis of a large series by Bosch, et al. [7], the optimal progesterone threshold over which a detrimental effect on IVF outcome might be observed has been estimated at $1.5 \mathrm{ng} / \mathrm{ml}$. Many studies reported a negative effect of PE during IVF cycles on the cycle outcomes including endometrial receptivity [1012]. Lower pregnancy rate and higher pregnancy loss with PE in IVF cycles had been observed in studies conducted by Silverberg, et al. [4], although the mechanism of this remains controversial [4,13]. Then, several authors investigated the effect of PE during the IVF cycles with GnRH analogues, some of these reported negative impact on the live birth and pregnancy rate, while others didn't find any association [14-18]. The aim of this study was to assess the validity of progesterone level on hCG day $\left(P_{4}\right)$ in the prediction of IVF/ ICSI cycles' outcome in a Saudi setting.

\section{Methods}

Our study was undertaken at King Abdul-Aziz Medical City of the National Guard- health affairs, department of obstetrics and gynecology, reproductive endocrinology and infertility unit, in Riyadh, Saudi Arabia. In a retrospective cohort study, all fresh cycles of patients who underwent IVF or ICSI cycles in 2017 with GnRH agonist or antagonist medications, were followed up. Frozen cycles were excluded. After reviewing all patients' files for infertility treatment at that period, 302 patients were eligible to be included in our study, 46 patients were cancelled for different reasons (21 patients had failed fertilization, 8 showed poor response, one developed ovarian hyperstimulation, 3 with no oocytes retrieved, one had an endometrial polyp, 6 had immature oocytes, and 6 had arrested embryos). Only 256 patients were followed up. Patients were started on antagonist [ $n=241]$, short [ $n=47]$ or long [ $n=14$ ] agonist protocols, according to their infertility evaluation. They were started on gonadotrophin medications for ovarian hyperstimulation. The starting dose was based on patient's age, AFC, BMI and previous response. The stimulation cycle was followed by serial transvaginal U/S and serum estrogen level $\left(E_{2}\right)$, each visit. When a patient had 2 or more $18 \mathrm{~mm}$ follicles, she was triggered by hCG 10,000 IU and ovum pickup was done 34-36 hrs after. We documented all patients' characteristics at the starting of the cycles. Number of follicles and endometrium thickness on hCG triggering day were also documented. We checked Progesterone level on the day of triggering $\left(P_{4}\right)$ as well as the estrogen level $\left(E_{2}\right)$.

Pregnancy rate was calculated. Among patients with positive pregnancy test, we calculated the rates of term live birth, preterm birth, abortion and chemical pregnancy. Patient's data and cycles' details for all patients were reviewed using patients' files and the EHR (Best-Care).

All values of the progesterone on the hCG day of IVF/ICSI cycles were cross classified according to their pregnancy test result (negative or positive), and by various cut-off points along with the range of progesterone levels above which subjects may be considered having a negative pregnancy test. From these tabulations, the sensitivity, specificity and positive predictive value were computed for progesterone level at each cut-off point.

The sensitivity of progesterone level diagnosis for the pregnancy test result "gold standard" was determined by calculating how frequent the correct progesterone level diagnosis was made in each pregnancy test diagnosis. The specificity of progesterone diagnosis was determined by calculating how frequently the progesterone diagnosis was not made when the corresponding pregnancy test diagnosis was positive. Positive predictability indicated how frequently the progesterone diagnosis correctly reflected the negative pregnancy test. Negative predictability indicated how frequently the progesterone diagnosis was not made when the corresponding pregnancy test diagnosis was positive. Also, the level of agreement between these two methods was determined at each cut-off point by the calculation of kappa coefficient $(k)$. Absolute risk reduction (ARR) and relative risk reduction (RRR) were calculated.

The Receiver Operating Characteristic (ROC) curve of a diagnostic test is a graph of the pairs of sensitivity and 1 minus specificity that correspond to each possible cutoff for the diagnostic test result. This curve was used to determine the threshold value of $P_{4}$ that corresponds to the negative pregnancy test result. Analyses were performed using SPSS (version 23). Both descriptive and analytical statistics were applied. Rates of pregnancy, abortion, live births were estimated. Chi-square test and Fisher exact were used to investigate the association between the levels of progesterone and outcome parameters. Logistic regression analysis was used to investigate if the change in progesterone level is a predictor of outcome. Significance was considered at the $p$ - value of $\leq 0.05$. The study was approved by the IRB of the Ministry of National Guard-Health Affairs (Ref. \# RC17/335/R). This study was conducted in accordance with the Declaration of Helsinki. 


\section{Results}

Of a total of 302 cycles, 46 cycles (15.2\%) were cancelled. The pregnancy rate in the followed up cycles $(n=256)$ was $36.7 \%$. There was a significant negative association between progesterone level at HCG day and the pregnancy outcome, with significantly higher mean progesterone level among those with a negative pregnancy test than its counterpart among those with a positive test $(2.26 \pm 1.81 \mathrm{vs} 1.84 \pm 1.01, t$ $=2.37, p=0.018$, Table 1 ).

Applying the ROC curve for progesterone levels on hCG day and the pregnancy test results shows that the optimum trade-off level for progesterone was $1.5 \mathrm{nmol} / \mathrm{L}$, figure 1 . This cut-off level had a sensitivity of $59 \%$ and a specificity of $51 \%$ for prediction of a negative pregnancy test. The computed positive predictive value was $68 \%$. The area under the curve (AUC) was 0.55 . This reflects the low validity of this cut-off in the prediction of pregnancy test results. At this cut-off level,

Table 1: 2 X 2 Cross tabulation of progesterone levels on hCG day and results of pregnancy test.

\begin{tabular}{|c|c|c|c|}
\hline & \multicolumn{2}{|c|}{ Pregnancy test $(\mathrm{n}=\mathbf{2 5 6})$} \\
\cline { 2 - 4 } & Negative $\mathrm{n}=162$ & Positive $\mathrm{n}=94$ & \\
\hline $\begin{array}{c}\text { Mean progesterone level on hCG } \\
\text { day (nmol/L) }\end{array}$ & $2.26 \pm 1.81$ & $1.84 \pm 1.01$ & $\begin{array}{c}t=2.37 \\
p=0.018\end{array}$ \\
\hline Progesterone level on hCG day & & & \\
\hline$\geq 1.5 \mathrm{nmol} / \mathrm{L}(\mathrm{n}, \%)$ & 96 & 46 & 142 \\
\hline$<1.5 \mathrm{nmol} / \mathrm{L}(\mathrm{n}, \%)$ & 66 & 48 & 114 \\
\hline
\end{tabular}

Sensitivity $=96 / 162=59 \%$; Specificity $=48 / 94=51 \%$; Positive Predictive Value (PPV) $=96 / 142=68 \%$, kappa $=0.103(p=0.077)$, AUC $=0.55$, Absolute Risk Reduction $($ ARR $)=(96 / 142)-(66 / 114)=10 \%$, Relative Risk Reduction $(R R R)=[(96 / 142)-$ $(66 / 114)] /(96 / 142)=15 \%$. the absolute risk reduction was $10 \%$ and the relative risk reduction was $15 \%$, i.e.; pregnancy failure would be reduced by $15 \%$ when $P_{4}$ level is $<1.5 \mathrm{nmol} / \mathrm{L}$. At this cut-off, the level of agreement between the $P_{4}$ level and the pregnancy test results, as calculated by kappa coefficient, was not significant ( $k=0.10, p=0.077)$, Table 1 .

Table 2 shows the comparison between cycles with $<1.5 \mathrm{nmol} / \mathrm{L}$ and $\geq 1.5 \mathrm{nmol} / \mathrm{L}$ mean progesterone level on the hCG day $\left(P_{4}\right)$, in terms of; patients characteristics, cycle characteristics, cycle outcome and complications. Cycles whose mean level of progesterone at hCG day is $\geq 1.5 \mathrm{nmol} / \mathrm{L}$ showed significantly higher proportion of primary infertility (54.1\% vs 39.2\%, $\chi 2=6.54, p=0.011)$, lower mean BMI (28.13 \pm 5.6 vs $29.76 \pm 4.9, t=2.62, p=0.009)$ and higher mean estrogen level (9602.09 \pm 6952 vs $5518.36 \pm 4359, t=6.22$, $p<0.001$ ). They showed also less favorable outcome in terms of term lower proportion of live birth (47.8\% versus $68.1 \%$, $\chi 2=3.92, p=0.048)$, higher proportion of abortion $(24.4 \%$ versus $8.2 \%, \chi 2=4.25, p=0.039)$, and higher proportion of ovarian hyperstimulation $(8.1 \%$ vs $2.3 \%, \chi 2=7.22, p=$ $0.027)$. Endometrial polyp was seen only in patients with low progesterone level. The incidence of premature progesterone elevation (PE) was $55.4 \%$ in all stimulation protocols. It was $58.5 \%(141 / 241)$ in antagonist protocol group, and $50.8 \%$ $(31 / 61)$ in agonist protocol group $(\chi 2=1.17, p=0.28)$.

After adjusting for BMI, type of infertility and estrogen level at hCG day, the significant negative association between $P_{4}$ and pregnancy outcome was retained, with a $p$ - value of $<0.03$ (Table 3).

Table 2: Demographic profile, protocol, stimulation protocol and parameters and outcomes in IVF/ICSI cycles for two progesterone levels on the hCG day.

\begin{tabular}{|c|c|c|c|c|}
\hline & \multicolumn{2}{|c|}{ Progesterone level at hCG day } & \multirow[b]{2}{*}{$x 2 / t$} & \multirow[b]{2}{*}{$P$ - value } \\
\hline & $<1.5 \mathrm{nmol} / \mathrm{L}(\mathrm{n}=114)$ & $\geq 1.5 \mathrm{nmol} / \mathrm{L}(\mathrm{n}=142)$ & & \\
\hline \multicolumn{5}{|c|}{ Patient characteristics } \\
\hline Age (mean $\pm \mathrm{SD})$ & $32.97 \pm 4.62$ & $32.43 \pm 4.7$ & $t=0.995$ & 0.322 \\
\hline $\begin{array}{c}\text { Type of infertility (no, \%) } \\
\text { Primary } \\
\text { Secondary }\end{array}$ & $\begin{array}{l}39.2 \%(51) \\
60.8 \%(79)\end{array}$ & $\begin{array}{l}54.1 \%(93) \\
45.9 \%(79)\end{array}$ & $\chi 2=6.54$ & 0.011 \\
\hline $\mathrm{BMI}($ mean $\pm \mathrm{SD})$ & $29.76 \pm 4.9$ & $28.13 \pm 5.6$ & $t=2.62$ & 0.009 \\
\hline \multicolumn{5}{|c|}{ Protocol \& Stimulation parameters } \\
\hline \multicolumn{5}{|l|}{ Protocol: } \\
\hline GnRH agonist $(20.2 \%, n=61)$ & $49.2 \%(30)$ & $50.8 \%(31)$ & & \\
\hline GnRH antagonist $(79.8 \%, n=241)$ & $41.5 \%(100)$ & $58.5 \%(141)$ & $\chi 2=1.17$ & 0.28 \\
\hline Endometrium thickness on hCG day & $12.24 \mathrm{~mm} \pm 13.6$ & $10.93 \mathrm{~mm} \pm 10.8$ & $t=0.915$ & 0.341 \\
\hline E2 level on HCG (mean \pm SD) & $5518.36 \pm 4359$ & $9602.09 \pm 6952$ & $t=6.22$ & $<0.001$ \\
\hline Number of Oocytes retrieved & $10.85 \pm 16.7$ & $13.46 \pm 17.8$ & $t=1.304$ & 1.97 \\
\hline \multicolumn{5}{|c|}{ Cycle's outcomes } \\
\hline & $\%$ (no.) & $\%$ (no.) & & $p$ - value \\
\hline Pregnancy rate $(36.7 \%, \mathrm{n}=94)$ & $42.6 \%(49)$ & $31.9 \%(45)$ & $\chi 2=3.12$ & 0.0 .77 \\
\hline Term Live birth $(58 \%, \mathrm{n}=54)^{\star}$ & $72.3 \%(34)$ & $46.7 \%(21)$ & $\chi 2=3.92$ & 0.048 \\
\hline Preterm live birth $(10.8 \%, 10)^{*}$ & $8.2 \%(4)$ & $13.3 \%(6)$ & - & $0.52^{@}$ \\
\hline Abortion $16.1 \%(n=15)^{*}$ & $8.2 \%(4)$ & $24.4 \%(11)$ & $\chi 2=4.25$ & 0.039 \\
\hline Chemical (no,\%): $(15.1 \%, 14)^{\star}$ & $14.3 \%(7)$ & $15.6 \%(7)$ & $\chi 2=0.002$ & 0.97 \\
\hline Cancelled cycles $(15.2 \%, n=46)$ & $11.5(15)$ & $18 \%(31)$ & & \\
\hline Cycle's Complications & \% (no.) & $\%$ (no.) & & \\
\hline Ovarian hyperstimulation $(5.6 \%, 17)$ & $2.3 \%(3)$ & $8.1 \%(14)$ & $\chi 2=7.22$ & 0.027 \\
\hline Endometrial polyp $(0.7 \%, 2)$ & $1.6 \%(2)$ & $0 \%(0)$ & - & - \\
\hline
\end{tabular}




\begin{tabular}{|c|c|c|}
\hline Independent variables & $B$ (SE) & $p$ - value \\
\hline Progesterone level at hCG day & $-0.24(0.11)$ & $<0.03^{\star \star}$ \\
\hline BMI & $-0.002(0.01)$ & 0.80 \\
\hline Infertility type (1ry vs 2ry) & $-0.008(0.26)$ & 0.98 \\
\hline Estrogen level at hCG day & $0.01(0.01)$ & 0.86 \\
\hline
\end{tabular}

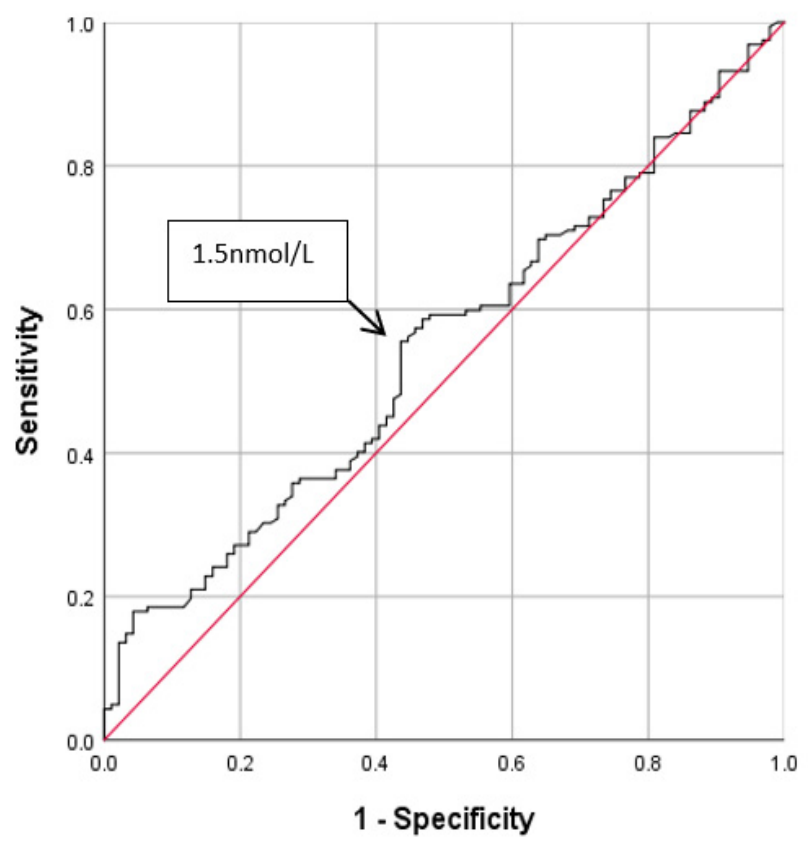

Figure 1: Receiver operating characteristic curve of cut-off of progesterone level at hCG day for pregnancy outcome.

\section{Discussion}

Pregnancy rate in our study was $36.7 \%$. This figure was comparable to figures of $30.6 \%$ [19] and 34\% [20] in previous studies. A progesterone rise during the late follicular phase $\left(P_{4}\right)$ has been considered a negative predictive factor for clinical outcome in both GnRH agonist [4,21] and antagonist protocols $[7,22]$. In our study, there was a significant negative association between $P_{4}$ level on hCG day and the pregnancy outcome, with significantly higher mean $P_{4}$ level among those with negative pregnancy test than its counterpart among those with positive test. This finding was in agreement with what was reported by Mascarenhas, et al. [20] and Ashmita, et al. [19], Higher $P_{4}$ level in our study was also associated with lower rate of term live birth and higher rates of abortion and ovarian hyperstimulation. Huang, et al. [17], in a study of 2566 patients, reported that PE negatively correlated with live birth in fresh embryo transfer cycles. Data from large previous retrospective [7] and prospective [23] studies supported the notion that pregnancy rates are inversely related to $P_{4}$ levels, especially when a threshold of $1.5 \mathrm{ng} / \mathrm{ml}$ is adopted.

The relationship between progesterone elevation (PE) and pregnancy rate has been analyzed by using different thresholds of serum progesterone on the day of hCG. The thresholds were varied and found to be between $0.4 \mathrm{ng} / \mathrm{ml}$ and
$3 \mathrm{ng} / \mathrm{ml}$ [9]. The analysis of a large series by Bosch, etal. [7], the optimal progesterone threshold over which a detrimental effect on IVF outcome might be observed has been estimated at $1.5 \mathrm{ng} / \mathrm{ml}$. In our study, applying the ROC curve for $P_{4}$ and the pregnancy test results showed that the optimum trade-off level for progesterone was $1.5 \mathrm{nmol} / \mathrm{L}$. This cut-off level had a sensitivity of $59 \%$ and a specificity of $51 \%$ for prediction of a pregnancy test result. The computed positive predictive value was $68 \%$. Thus, at this cut-off level, the progesterone test correctly diagnosed $59 \%$ of negative pregnancy test results, missed $41 \%$ of these negative pregnancy tests, but misclassified $49 \%$ of positive pregnancy results as negative results (false positives). At this cut-off level, absolute risk reduction was $10 \%$ and relative risk reduction was $15 \%$, i.e.; pregnancy failure would be reduced by $15 \%$ when the level of $P_{4}$ is less than $1.5 \mathrm{nmol} / \mathrm{L}$. The level of agreement between the $P_{4}$ and the pregnancy test results, as calculated by kappa coefficient, was not significant $(k=0.10, p=0.077$ ). Meanwhile, there was no significant difference between the group with $P_{4}$ $<1.5 \mathrm{nmol} / \mathrm{L}$ and those with $\geq 1.5 \mathrm{nmol} / \mathrm{L}$ in pregnancy rate. These findings may reflect that the low validity of the cut-off of $1.5 \mathrm{nmol} / \mathrm{L}$ for $P_{4}$ in the prediction of pregnancy outcome. Even, using the cut-off of $\geq 1.2 \mathrm{nmol} / \mathrm{L}$, recommended by others [7], would result in a very low (28\%) sensitivity, 69\% specificity and $61 \%$ positive predictive value.

The pathogenesis of PE in controlled ovarian hyperstimulation $(\mathrm{COH})$ cycles is still poorly understood. The incidence of PER in our study was 55.5\% (142/256), based on a cut-off level of $1.5 \mathrm{nmol} / \mathrm{L}$ for $P_{4}$. This figure is high if compared with figures of $13.19 \%$ [19], 13.02\% [18] and $38.3 \%$ [7]. However, comparison is difficult when the definition of PE is different in various studies. Among factors associated with PE are the type of protocol, the type and total dose of gonadotropin given, $E_{2}$ levels on the day of trigger and the number of intermediate follicles recruited [19]. Bosch, et al. [7] concluded that estrogen values on the day of hCG trigger were associated with increased progesterone levels $(P$ $<0.0001$ ).In our study, higher mean level of $P_{4}$ of $\geq 1.5 \mathrm{nmol} / \mathrm{L}$ showed significantly higher mean estrogen level $\left(E_{2}\right)$. This was in agreement with the findings of Ashmita, et al. [19] who reported a higher incidence of PE in the cycles with $E_{2} \geq 2500$ IU. The incidence of elevated progesterone concentrations was higher in rFSH-treated patients than in HMG-treated patients [23], and in cases with large doses of gonadotropins given $[19,24]$. However, in our study, there was no significant association between the type of protocol and $P_{4}$ level. The mean number of oocytes retrieved was significantly higher in patients with higher $P_{4}$ levels [25], but our study showed no such association. In our study, significant associations were shown between $P_{4}$ level and only 3 variables; type of infertility and BMI and $E_{2}$ level, however, none of these variables had a significant association with the pregnancy rate. Adjusting for these variables, $P_{4}$ level was the only significant predictor of pregnancy outcome. 


\section{Conclusion}

Premature progesterone level on the day of hCG in IVF/ ICSI cycles might have a role in predicting pregnancy outcome. However, the cut-off of $\geq 1.5 \mathrm{nmol} / \mathrm{L}$ for progesterone on the hCG day is not a valid threshold in the prediction of pregnancy outcome. Further studies are necessary to confirm these findings.

\section{Declarations}

\section{Ethics approval and consent to participate}

This study was approved by the institutional review board (IRB) of the Ministry of National Guard-Health Affairs, Riyadh, Saudi Arabia [Ref. \# RC17/335/R]. The need for informed consent was waived by the IRB, as all these retrospective data were retrieved from records without the disclosure of identifiers.

\section{Availability of data and materials}

The datasets used and/or analyzed during the current study are available from the corresponding author on reasonable request.

\section{Author contributions}

HA, SH and MAA contributed to concept development, manuscript preparation and final writing, $\mathrm{HA}$ and $\mathrm{SH}$ contributed to concept development, research proposal writing and data collection, MAA and HA conducted data analysis and interpretation, and manuscript drafting. All authors read and approved the final manuscript.

\section{Acknowledgement}

This study was initiated and supported by King Abdullah International Medical Research Center (KAIMRC), King Saud bin-Abdulaziz University for Health Sciences, Ministry of National Guard -Health Affairs, Saudi Arabia. The final draft of the manuscript was English language edited by Macmillan Science Communication.

\section{References}

1. Hugues JN, Massé-Laroche E, Reboul-Marty J, Boîko O, Meynant $C$, et al. Impact of endogenous luteinizing hormone serum levels on progesterone elevation on the day of human chorionic gonadotropin administration. Fertil Steril. 2011; 96: 600-604.

PubMed: https://www.ncbi.nlm.nih.gov/pubmed/21880277

2. Del Castillo JL, Bousamra M, De La Fuente L, Ruiz-Balda JA, Palomo $M$. The impact of serum progesterone levels on the results of in vitro fertilization treatments: a literature review. JBRA Assist Reprod. 2015; 19: 141-147.

PubMed: https://www.ncbi.nlm.nih.gov/pubmed/27203093

3. Edelstein MC, Seltman HJ, Cox BJ, Robinson SM, Shaw RA, et al Progesterone levels on the day of human chorionic gonadotropin administration in cycles with gonadotropin-releasing hormone agonist suppression are not predictive of pregnancy outcome. Fertil Steril. 1990; 54: 853-857.

PubMed: https://www.ncbi.nlm.nih.gov/pubmed/2121554
4. Silverberg KM, Burns WN, Olive DI, Riehl RM, Schenken RS. Serum progesterone levels predict success of in vitro fertilization/embryo transfer in patients stimulated with leuprolide acetate and human menopausal gonadotropins. J Clin Endocrinol Metab. 1991; 73:797-803. PubMed: https://www.ncbi.nlm.nih.gov/pubmed/1909704

5. Bosch E, Valencia I, Escudero E, Crespo J, Simón C, et al. Premature luteinization during gonadotropin-releasing hormone antagonist cycles and its relationship with in vitro fertilization outcome. Fertil Steril. 2003; 80: 1444-1449.

PubMed: https://www.ncbi.nlm.nih.gov/pubmed/14667881

6. Ubaldi $F$, Albano $C$, Peukert $M$, Riethmüller-Winzen $H$, Camus $M$, et al. Endocrinology: subtle progesterone rise after the administration of the gonadotrophin-releasing hormone antagonist Cetrorelix in intracytoplasmic sperm injection cycles. Hum Reprod. 1996; 11: 14051407.

PubMed: https://www.ncbi.nlm.nih.gov/pubmed/8671476

7. Bosch E, Labarta E, Crespo J, Simon C, Remohi J, et al. Circulating progesterone levels and ongoing pregnancy rates in controlled ovarian stimulation cycles for in vitro fertilization: analysis of over 4000 cycles. Human reproduction. 2010; 25: 2092-2100.

PubMed: https://www.ncbi.nlm.nih.gov/pubmed/20539042

8. Al-Azemi M, Kyrou D, Kolibianakis EM, Humaidan P, Van Vaerenbergh I, et al. Elevated progesterone during ovarian stimulation for IVF. Reprod Biomed Online. 2012; 24: 381-388.

PubMed: https://www.ncbi.nlm.nih.gov/pubmed/22377153

9. Venetis CA, Kolibianakis EM, Bosdou JK, Tarlatzis BC. Progesterone elevation and probability of pregnancy after IVF: a systematic review and meta-analysis of over 60000 cycles. Hum Reprod Update. 2013; 19: 433-457.

PubMed: https://www.ncbi.nlm.nih.gov/pubmed/23827986

10. Labarta E, Martínez-Conejero JA, Alamá P, Horcajadas JA, Pellicer A, et al. Endometrial receptivity is affected in women with high circulating progesterone levels at the end of the follicular phase: a functional genomics analysis. Hum Reprod. 2011; 26: 1813-1825.

PubMed: https://www.ncbi.nlm.nih.gov/pubmed/21540246

11. Li R, Qiao J, Wang L, Li L, Zhen X, et al. MicroRNA array and microarray evaluation of endometrial receptivity in patients with high serum progesterone levels on the day of hCG administration. Reprod Biol Endocrinol. 2011; 9: 29 .

PubMed: https://www.ncbi.nlm.nih.gov/pubmed/21375772

12. Haouzi D, Bissonnette L, Gala A, Assou S, Entezami F, et al. Endometrial receptivity profile in patients with premature progesterone elevation on the day of HCG administration. BioMed Res Int. 2014; 2014.

PubMed: https://www.ncbi.nlm.nih.gov/pmc/articles/PMC4022194/

13. Schoolcraft W, Sinton E, Schlenker T, Huynh D, Hamilton F, et al Lower pregnancy rate with premature luteinization during pituitary suppression with leuprolide acetate. Fertil Steril. 1991; 55: 563-566. PubMed: https://www.ncbi.nlm.nih.gov/pubmed/1900481

14. Xu B, Li Z, Zhang H, Jin L, Li Y, et al. Serum progesterone level effects on the outcome of in vitro fertilization in patients with different ovarian response: an analysis of more than 10,000 cycles. Fertil Steril. 2012; 97: 1321-1327.

PubMed: https://www.ncbi.nlm.nih.gov/pubmed/22494924

15. Papanikolaou EG, Pados G, Grimbizis G, Bili E, Kyriazi L, et al. GnRHagonist versus $\mathrm{GnRH}$-antagonist IVF cycles: is the reproductive outcome affected by the incidence of progesterone elevation on the day of HCG triggering? A randomized prospective study. Hum Reprod. 2012; 27: 1822-1828.

PubMed: https://www.ncbi.nlm.nih.gov/pubmed/22422777

16. Ochsenkühn R, Arzberger A, von Schönfeldt V, Gallwas J, Rogenhofer $\mathrm{N}$, et al. Subtle progesterone rise on the day of human chorionic 
gonadotropin administration is associated with lower live birth rates in women undergoing assisted reproductive technology: a retrospective study with 2,555 fresh embryo transfers. Fertil Steril. 2012; 98: 347-354. PubMed: https://www.ncbi.nlm.nih.gov/pubmed/22633265

17. Huang CC, Lien YR, Chen HF, Chen MJ, Shieh CJ, et al. The duration of pre-ovulatory serum progesterone elevation before $\mathrm{hCG}$ administration affectstheoutcomeofIVF/ICSIcycles. HumReprod.2012;27:2036-2045 PubMed: https://www.ncbi.nlm.nih.gov/pubmed/22561057

18. Huang PC, Chen MJ, Guu HF, Yi YC, Ho JY, et al. Effect of premature serum progesterone rise on embryo transfer outcomes and the role of blastocyst culture and transfer in assisted reproductive technology cycles with premature progesterone rise. Taiwan J Obstet Gynecol. 2015; 54: 641-646.

PubMed: https://www.ncbi.nlm.nih.gov/pubmed/26700978

19. Ashmita J, Vikas S, Swati G. The impact of progesterone level on day of hCG injection in IVF Cycles on clinical pregnancy rate. J Hum Reprod Sci. 2017; 10: 265-270.

PubMed: https://www.ncbi.nlm.nih.gov/pubmed/29430153

20. Mascarenhas M, Kamath MS, Chandy A, Kunjummen AT. Progesterone/ estradiol ratio as a predictor in the ART cycles with premature progesterone elevation on the day of hCG trigger. J Reprod Infertil. 2015; 16: 155-161.

PubMed: https://www.ncbi.nlm.nih.gov/pubmed/26913234
21. Elnashar AM. Progesterone rise on the day of HCG administration (premature luteinization) in IVF: an overdue update. J Assist Reprod Genet. 2010; 27: 149-155.

PubMed: https://www.ncbi.nlm.nih.gov/pubmed/20177771

22. Papanikolaou EG, Kolibianakis EM, Pozzobon C, Tank P, Tournaye H, et al. Progesterone rise on the day of human chorionic gonadotropin administration impairs pregnancy outcome in day 3 single-embryo transfer, while has no effect on day 5 single blastocyst transfer. Fertil Steril. 2009; 91: 949-952.

PubMed: https://www.ncbi.nlm.nih.gov/pubmed/17555751

23. Andersen AN, Devroey P, Arce JC. Clinical outcome following stimulation with highly purified hMG or recombinant FSH in patients undergoing IVF: a randomized assessor-blind controlled trial. Human Reproduction. 2006; 21: 3217-3227.

PubMed: https://www.ncbi.nlm.nih.gov/pubmed/16873892

24. KiliçdagEB,HaydardedeogluB,CokT,HaciveliogluSO,BagisT.Premature progesterone elevation impairs implantation and live birth rates in $\mathrm{GnRH}$ agonist IVF/ICSI cycles. Arch Gynecol Obstet. 2010; 281: 747-752. |PubMed: https://www.ncbi.nlm.nih.gov/pubmed/19862542

25. Kyrou D, Al-Azemi M, Papanikolaou EG, Donoso P, Tziomalos $\mathrm{K}$, et al The relationship of premature progesterone rise with serum estradiol levels and number of follicles in GnRH antagonist/recombinant FSHstimulated cycles. Eur J Obstet Gynecol Reprod Biol. 2012; 162:165-168. PubMed: https://www.ncbi.nlm.nih.gov/pubmed/22425288 